\title{
Differences in lipopolysaccharide profiles of serologically identical Legionella pneumophila serogroup 6 strains
}

\author{
O G ZANEN-LIM, H C ZANEN
}

From the Department of Medical Microbiology, Academic Medical Centre, University of Amsterdam, Amsterdam, The Netherlands

SUMMARY Over five years 18 strains of Legionella pneumophila serogroup 6 were isolated in Amsterdam from the hot water supply in three hospitals and from one patient. Immunodiffusion and immunoblot procedures showed that these strains were identical. Profiles of isolated lipopolysaccharides from the 18 strains and the reference serogroup 6 strain were visualised in polyacrylamide gels stained with silver. Four strains from hospital A, isolated in 1982, 1984, and 1985 displayed similar lipopolysaccharide profiles which were different in relative mobility from those of hospitals B and C. Those from hospital B (12 strains isolated in 1983 and 1986) and C (one strain) were similar in relative mobility but different in colour. The strain from a patient with acquired immune deficiency syndrome (AIDS) in hospital A displayed a lipopolysaccharide profile characteristic of hospital A. These reproducible profiles were all different in relative mobility from the reference serogroup 6 strain. They can be used as a marker system in epidemiological surveys of serologically identical serogroup 6 strains. Lipopolysaccharide patterns from strains isolated throughout the years in the same hospital were similar. This suggests an outgrowth from organisms inhabiting the plumbing system rather than reseeding from the Amsterdam mains supply.

Outbreaks of legionellosis in hospitals have often been associated with the presence of legionellae in the hot water supply. ${ }^{12}$ Raising the temperature of the hot water prevented nosocomial infections but did not eradicate the organisms. ${ }^{34}$ In a university hospital in Amsterdam Legionella pneumopila serogroups 1 and 6 were isolated from hot water taps which were the source of infections from serogroup $1 .{ }^{5}$ After raising the temperature of the hot water in April 1982 four nosocomial infections occurred due to serogroup 1. When serogroup 6 was isolated for the first time from a patient's sputum in November 1985, the possibility of a nosocomial infection was considered. We therefore investigated all the available environmental serogroup 6 strains isolated in Amsterdam in three hospitals, together with the patient's strain.

\section{Material and methods}

Strains were isolated from hot tapwater in three hospitals connected to the municipal water supply of Amsterdam. Hospital A is a university hospital,

Accepted for publication 17 March 1988 located southeast of Amsterdam; hospital B is situated near the centre; and hospital $C$ is a university hospital in the western part of the city. Because of legionellosis occurring in hospital A, water samples from hot water taps and shower heads were investigated for the presence of legionellae. Strains were isolated, identified, and serogrouped as described previously ${ }^{5}$ and kept at $-70^{\circ} \mathrm{C}$ in peptone-glycerol. L pneumophila serogroup 1, isolated from taps on all floors of the building, caused nosocomial illness as previously described. ${ }^{5}$ Serogroup 6 was isolated sporadically and did not cause nosocomial disease. After raising the water temperature to $60^{\circ} \mathrm{C}$ at the outlet of the taps serogroups 1 and 6 were cultured only occasionally and in small numbers. When taps were used infrequently or the required temperature was not reached, however, legionellae were isolated in greater numbers and gave rise to four nosocomial infections due to serogroup 1. When serogroup 6 was isolated from a patient with AIDS who developed pneumonia in November 1985, all serogroup 6 strains stored at $-70^{\circ} \mathrm{C}$ were investigated. They comprised strains isolated in hospital A from hot tapwater during routine sampling (one in September 1982, two in 
September 1984, one in July 1985), strains sent in by hospital B (10 strains isolated in 1983 and two in 1986), and by hospital C (one strain). They were studied together with the reference serogroup 6 strain (Chicago 2).

\section{DOUBLE DIFFUSION PROCEDURE}

This was done as described previously ${ }^{5}$ with antiserum raised in rabbits against the reference serogroup 6 strain and antigens in the supernatants of boiled cell suspensions of the strains. Briefly, glass slides $(5 \times 5)$, covered with a $1 \mathrm{~mm}$ layer of a solution containing $1.5 \%$ special agar Noble (Difco, Detroit) in phosphate buffered saline (PBS) (pH 7.2), were punched with holes $2 \mathrm{~mm}$ in diameter and $3 \mathrm{~mm}$ apart and filled to the rim. After incubation in a moist atmosphere for 24 to 48 hours at room temperature the slides were washed in PBS, dried, and stained with Coomassie brilliant blue.

\section{EXTRACTION OF LIPOPOLYSACCHARIDE}

Lipopolysaccharides were extracted as described in detail in a previous publication. ${ }^{6}$ Briefly, $50 \mu \mathrm{l}$ of the clear supernatant of a boiled cell suspension was heated at $100^{\circ} \mathrm{C}$ for 10 minutes with $50 \mu \mathrm{l}$ buffer $(4 \%$ sodium dodecyl sulphate, $10 \%$ 2-mercaptoethanol, $20 \%$ glycerol in $0.625 \mathrm{M}$ Tris hydrochloric acid ( $\mathrm{pH}$ 6.8 ) and bromophenol blue). After incubation with $20 \mu \mathrm{l}$ proteinase $\mathrm{K}(2.5 \mathrm{mg} / \mathrm{ml}$ of the above buffer) lipopolysaccharide was precipitated twice from the supernatant with two volumes of $0.375 \mathrm{M} \mathrm{MgCl}_{2}$ in $95 \%$ ethanol. The resulting pellets were suspended in distilled water and frozen at $-20^{\circ} \mathrm{C}$. Lipopolysaccharide was checked for purity in polyacrylamide gels with protein (Coomassie brilliant blue) and silver stains. To assess reproducibility isolations of lipopolysaccharides were done three times.

\section{ELECTROBLOTTING}

The procedure of Laemmli $^{7}$ was used as described before $^{6}$ for electrophoresis of lipopolysaccharide preparations. The method of Pyle and Schill with modifications $^{8}$ was used for electroblotting. Polyacrylamide gels with resolved lipopolysaccharide bands were immediately transferred to $200 \mathrm{ml}$ of transfer buffer ( $25 \mathrm{mM}$ Tris and $192 \mathrm{mM}$ glycine, $\mathrm{pH}$ 8.3) and equilibrated for 15 minutes. Electrophoretic transfer conditions were three hours at 55 volts, cooled at $4^{\circ} \mathrm{C}$ in a Bio-Rad transblot apparatus (Bio-Rad Laboratories, Richmond, California). Immunodetection of lipopolysaccharides bound to nitrocellulose sheets (BA-85, 0.45 $\mu \mathrm{m}$, Schleicher and Schuell, Keene New Hampshire, USA) was done as follows: the sheets were washed in Tris-buffered saline (TBS: pH 7.5, 20 $\mathrm{mM}$ Tris and $500 \mathrm{mM}$ sodium chloride) for 10 minutes at room temperature, blocked in $3 \%$ gelatine TBS for
30 minutes, incubated overnight with primary antiserum diluted in $1 \%$ gelatine TBS, rinsed briefly in deionised water, and washed twice in TBS with $0.025 \%$ Tween 20 for 10 minutes, reacted for one hour in horseradish peroxidase labelled sheep anti-rabbit IgG diluted $1 / 1000$ in $1 \%$ gelatine TBS, washed twice with $0.025 \%$ Tween 20 in TBS for 10 minutes, and developed in a substrate solution containing $0.06 \% 4$ chloro-1-naphthol (Merck Sharpe and Dome) and $0.015 \% \mathrm{H}_{2} \mathrm{O}_{2}$. The stained blots were washed in distilled water, dried, and stored in the dark.

\section{Results}

The supernatants of boiled cell suspensions of the wild type and the reference serogroup 6 strains reacted with serogroup 6 specific rabbit antiserum, resulting in complete fusion of the precipitation lines in the double diffusion test. No reaction was left after adsorption with each of the wild type strains (data not shown). Blots of polyacrylamide gels with resolved lipopolysaccharides of the strains were reacted with

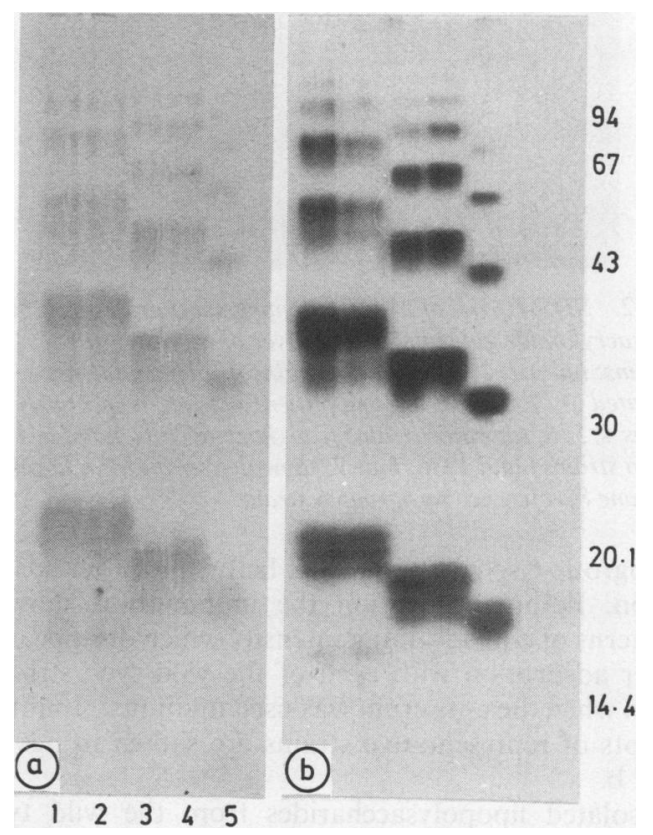

Fig 1 (a) Immunoblot patterns of isolated lipopolysaccharides of representative serogroup 6 strains. with serogroup 6 specific rabbit antiserum used as second antibody. Lane 1, tapwater strain from hospital C: lane 2. tapwater strain from hospital B; lanes 3 and 4, tapwater strains from hospital $A$ isolated in 1982 and 1984: lane 5. reference serogroup 6 strain. (b) Polyacrylamide gel stained with silver after being blotted. Position and molecular weight of protein markers are indicated on the right. 


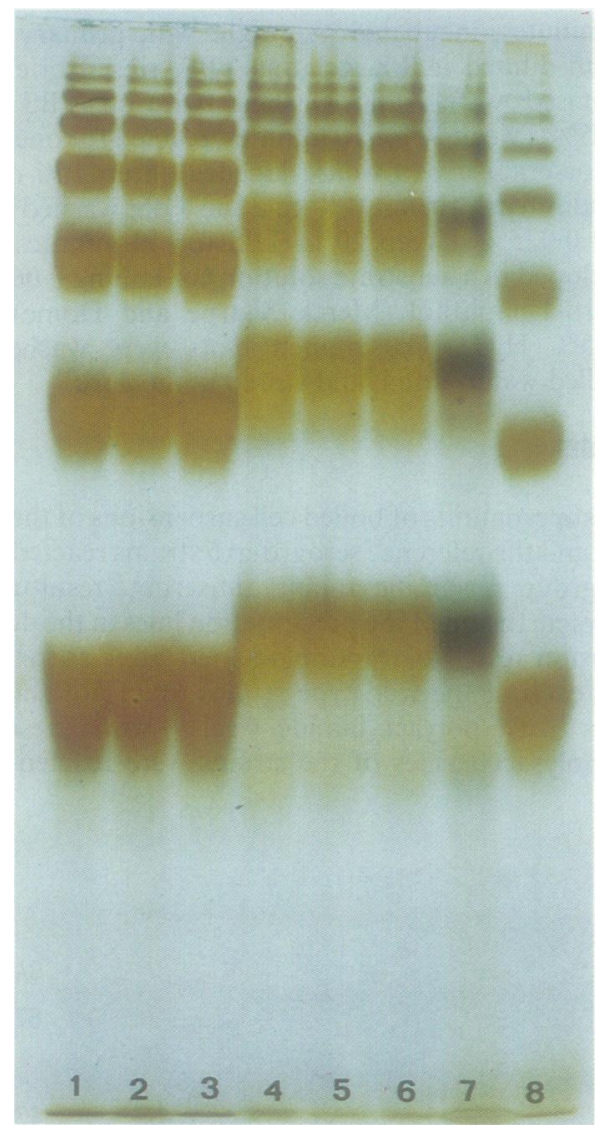

Fig 2 SDS-PAGE of isolated lipopolysaccharides in 12\% polyacrylamide gel stained with silver of representative strains: lanes 1, 2, 3, tapwater strains from hospital $A$ isolated in 1982 and 1985 and patient's strain, respectively; lanes 4, 5, 6, tapwater strains from hospital B isolated in 1983 (two strains) and 1986; lane 7, tapwater strain from hospital $C$; lane 8 , reference serogroup 6 strain.

serogroup 6 specific antiserum before and after adsorption. Before adsorption the immunoblot showed patterns of equal staining intensity which disappeared after adsorption with each of the wild type strains, even when the antiserum was used undiluted. Immunoblots of representative strains are shown in figs 1a and $b$.

Isolated lipopolysaccharides from the wild type and the reference serogroup 6 strains, resolved in polyacrylamide gels and stained with silver, displayed smooth type lipopolysaccharides, with high molecular weight repeating units (range 15 to 100 kilodaltons). Coomassie brilliant blue staining showed no protein staining. Four strains isolated from hot tapwater in September 1982 , September 1984 , July 1985 , and the patient's strain from hospital A showed lipopolysac- charide patterns similar in relative mobility and colour. Twelve strains isolated in hospital B from hot tapwater in 1983 and 1986 showed relative mobilities of lipopolysaccharide profiles which differed from those of hospital A, but resembled that of the strain in hospital $\mathrm{C}$. The colours of the lipopolysaccharide profiles of the strains from hospitals A and B were almost identical in colour (orange brown), but the strain of hospital $\mathrm{C}$ showed a lipopolysaccharide profile that was black-brown in colour. All the hospital strains displayed lipopolysaccharide profiles that differed in terms of relative mobilities from that of the reference serogroup 6 strain. Serogroup 6 strains from the same hospital showed similar lipopolysaccharide profiles throughout the years. These differences in relative mobilities and colour were reproducible when lipopolysaccharide isolations of all the strains were repeated three times with two to four week intervals. Doubling the quantity of lipopolysaccharides in the slots only increased the expression of the repeating units, but the differences in colour and relative mobility remained. Lipopolysaccharide profiles of representative strains are shown in fig 2 .

\section{Discussion}

$L$ pneumophila serogroup 6, first described by McKinney et al, ${ }^{9}$ has been implicated in several outbreaks of legionellosis subsequently confirmed by culture. ${ }^{10-12}$ In the United States serogroup 6 is second only to serogroup 1 as a cause of pneumonia due to $L$ pneumophila. ${ }^{13}$ Epidemiological associations between clinical and environmental isolates were mostly based on circumstantial evidence and on congruent serogroups. The ubiquity of $L$ pneumophila, however, implies that more precise characterisation of a legionella isolate is needed to permit specific tracing of an aetiological agent to its source. Plasmid analysis of serogroup 6 strains investigated so far has reported only the absence of plasmids. ${ }^{14}$ Monoclonal antibodies to serogroup 6 were raised, ${ }^{15-17}$ but these were all serogroup specific and not useful for subtyping. We also failed to differentiate wild type and reference serogroup 6 strains by the immunodiffusion procedure or by the sensitive immunoblot test, using polyclonal antibodies. These serologically identical strains, however, could be differentiated by their lipopolysaccharide profile. Isolated lipopolysaccharides, visualised in silver stained polyacrylamide gels, showed lipopolysaccharide profiles characteristic of the hospital in which they were isolated. These distinctive lipopolysaccharide patterns remained constant in the strains isolated in 1982, 1984, and 1985 in hospital A, in spite of storage and transfer of these strains more than 15 times on buffered charcoal yeast extract agar. The same applies to the strains isolated in 1983 and 
1986 in hospital B. The environmental strain from hospital $C$ could be differentiated from those of hospitals A and B by the black-brown colour of its lipopolysaccharide pattern; those of hospitals $A$ and B were orange-brown but not similar in relative mobility. The patient with AIDS was treated intermittently in hospital A from January 1985 until he died in March 1986. During this period he visited the premises where serogroup 6 was isolated from hot water taps at routine sampling in 1982 and 1985. In November 1985 he developed pneumonia due to $L$ pneumophila serogroup 6. Attempts to isolate legionellae from his ward and home did not yield any organisms, but the temperature from the hot water in his quarters was $58^{\circ} \mathrm{C}$. Lipopolysaccharide extracted from the strain cultured from his sputum showed a pattern typical of hospital $\mathrm{A}$, which strongly suggests a nosocomial infection.

The degree of susceptibility of hosts and the number of organisms of sufficient virulence needed to cause Legionnaires' disease, is not known. Although heating the tapwater to $60^{\circ} \mathrm{C}$ at the outlet significantly reduces the number of viable legionellae, eradication is not achieved. ${ }^{34}$ The unchanged lipopolysaccharide characteristics of the environmental serogroup 6 strains isolated throughout the years indicates the persistance of these strains in niches of the plumbing system rather than recolonisation from the mains supply. Apparently the ecological properties of the foci in these buildings are sufficiently constant to permit these organisms to survive in small numbers and to increase in number when control measures are relaxed.

We conclude that lipopolysaccharide typing of serologically identical serogroup 6 strains is useful in epidemiological surveys. The persistence of low numbers of legionellae in a colonised hospital water system necessitates continuous vigilance. Occasional infections occur, either because the susceptibility of patients increases or the virulence of micro-organisms changes.

We thank the laboratory staff for useful discussions and W Hersbach for excellent photography.

\section{References}

1 Tobin JO`H. Beare J, Dunnill MS, et al. Legionnaires' disease in a transplant unit: isolation of the causative agent from shower baths. Lancet 1980;ii:118-21.
2 Fraser DW. Potable water as a source for legionellosis. Environ Health Perspect 1985;62:337-41.

3 Plouffe JF, Webster LR, Hackman B. Relationship between colonisation of hospital buildings with Legionella pneumophila and hot water temperatures. Appl Environ Microhiol 1983;46:769-70.

4 Ribeiro CD, Burge SH, Palmer SR, Tobin JO'H, Watkins ID. Legionella pneumophila in a hospital water system following a nosocomial outbreak: prevalence, monoclonal antibody subgrouping and effect of control measures. Epidemiology and Infection 1987;98:253-62.

5 Zanen-Lim OG, Van den Broek NJ, Rietra PJGM, Zanen HC. Comparison of strains of Legionella pneumophila serogroup 1 isolated in four Amsterdam hospitals from patients and hot water supplies. $J$ Infect Dis 1984;150:508-12.

6 Zanen-Lim OG, Zanen HC. Lipopolysaccharides as a marker system in the epidemiology of Legionella pneumophila serogroup 12. FEMS Microhiol Lett 1988;50:85-8.

7 Laemmli UK. Cleavage of structural proteins during the assembly of the head of bacteriophage T4. Nature 1970;227:680-5.

8 Pyle SW, Schill WB. Rapid serological analysis of bacterial lipopolysaccharides by electrotransfer to nitrocellulose. J Immunol Methods 1985;85:371-82.

9 McKinney RM, Wilkinson HW, Sommers HM, et al. Legionella pneumophila serogroup six: isolation from cases of legionellosis. identification by immunofluorescence staining, and immunological response to infection. J Clin Microhiol 1980:12:395-401.

10 Cordes LG, Wiesenthal AM, Gorman GW, et al. Isolation of Legionella pneumophila from hospital shower heads. Ann Intern Med 1981;94:195-7.

11 Arnow PM, Chou T, Weil D. Shapiro EN, Kretzschmar C. Nosocomial Legionnaires' disease caused by aerosolized tap water from respiratory devices. J Infect Dis 1982;146:460-7.

12 Dowling JN, Pasculle AW, Frola FN, Zaphyr MK, Yee RB. Seroepidemiological study of legionella infections in renal transplant recipients. In: Thornsberry C, Balows A. Feeley JC, Jakubowski W, eds. Legionella: Proceedings of the Second International Symposium. Washington. DC: American Society for Microbiology, 1984:229-31.

13 Reingold AL, Thomason BM, Brake BJ, Thacker L, Wilkinson HW, Kuritsky JN. Legionella pneumonia in the United States: the distribution of serogroups and species causing human illness. J Infect Dis 1984;149:819.

14 Aye T. Wachsmuth K, Feeley JC, Gibson RJ, Johnson SR. Plasmid profiles of legionella species. Curr Microhiol 1981:6:389-94.

15 Para MF. Plouffe JF. Production of monoclonal antibodies to Legionella pneumophila serogroups 1 and 6. J Clin Microhiol 1983;18:895-900.

16 Joly JR, Chen Y, Ramsay D. Serogrouping and subtyping of Legionella pneumophila with monoclonal antibodies. J Clin Microhiol 1983:18:1040-6.

17 Watkins ID. Tobin JO'H. Studies with monoclonal antibodies to legionella species. In: Thornsberry C. Balows A, Feeley JC, Jakubowski W, eds. Legionella: Proceedings of the Second International Symposium. Washington, DC: American Society for Microbiology, 1984:259-62.

Requests for reprints to: Dr OG Zanen-Lim, Vreelandseweg 23, 3632 ER Loenen aan de Vecht, The Netherlands. 